Case

\title{
Left Coronary Ostial Stenosis after the Modified Bentall Using a Long Interposed Coronary Graft in a Patient with Pectus Excavatum
}

\author{
Yasuyuki Kato, MD, Koji Hattori, MD, Manabu Motoki, MD, Yosuke Takahashi, MD, \\ Shinsuke Nishimura, MD, and Toshihiko Shibata, MD
}

\begin{abstract}
A 26-year-old man presented chest oppression. He had pectus excavatum associated with Loeys-Dietz syndrome and a history of redo aortic root replacement with the modified Bentall technique using an 8-mm long interposed graft to the left coronary ostium. Coronary angiography revealed severe stenosis of both left coronary ostium and proximal left anterior descending artery, which was supposed to be resulted from thrombosis in the interposed graft. The left coronary system was bypassed through a left thoracotomy, which was suitable in this patient because the pectus excavatum would prevent harvest of the left internal thoracic artery through re-median sternotomy and to avoid potential sternal reentry injury of the heart. Although the left anterior descending artery was easily accessed under off-pump technique, exposure and anastomosis of the circumflex coronary artery was more difficult than expected without cardiopulmonary bypass as the pectus excavatum and adhesion of the heart prevented anterior shift and rotation of the heart.
\end{abstract}

Keywords: coronary ostial stenosis, bentall, left thoracotomy, pectus excavatum

\section{Introduction}

Anastomotic complication of the interposed graft after the Bentall procedure is a rare but life-threatening complication. We describe a case of severe stenosis between the long interposed graft and the left coronary ostium (LCO) after a redo Bentall using Svensson's modification. ${ }^{1)}$ This patient had pectus excavatum associated with Loeys-Dietz syndrome (LDS), and under-

$\overline{\text { Department of Cardiovascular Surgery, Osaka City General }}$ Hospital, Miyakojima-ku, Osaka, Japan

Received: September 24, 2012; Accepted: November 5, 2012 Corresponding author: Yasuyuki Kato, MD. Department of Cardiovascular Surgery, Osaka City General Hospital, 2-13-22, Miyakojima-hondori, Miyakojima-ku, Osaka 534-0021, Japan Email: kato20001224@gmail.com

(C)2014 The Editorial Committee of Annals of Thoracic and Cardiovascular Surgery. All rights reserved. went coronary artery bypass grafting (CABG) to the left coronary system through a left thoracotomy approach. Management of this case and possible causes of coronary ostial stenosis after the modified Bentall are discussed.

\section{Case Report}

A 26-year-old man was admitted to our hospital. He had been diagnosed with LDS and had pectus excavatum. At 16 years of age, he underwent aortic root replacement through a median sternotomy. Both coronary arteries were reconstructed by the button technique. At 23 years of age, redo aortic root replacement through re-median sternotomy was required for pseudoaneurysmal formation of the aortic root. The modified Bentall technique using an 8-mm interposed graft to the LCO was applied because the LCO was tightly bound to the surrounding tissue. The right coronary artery was 


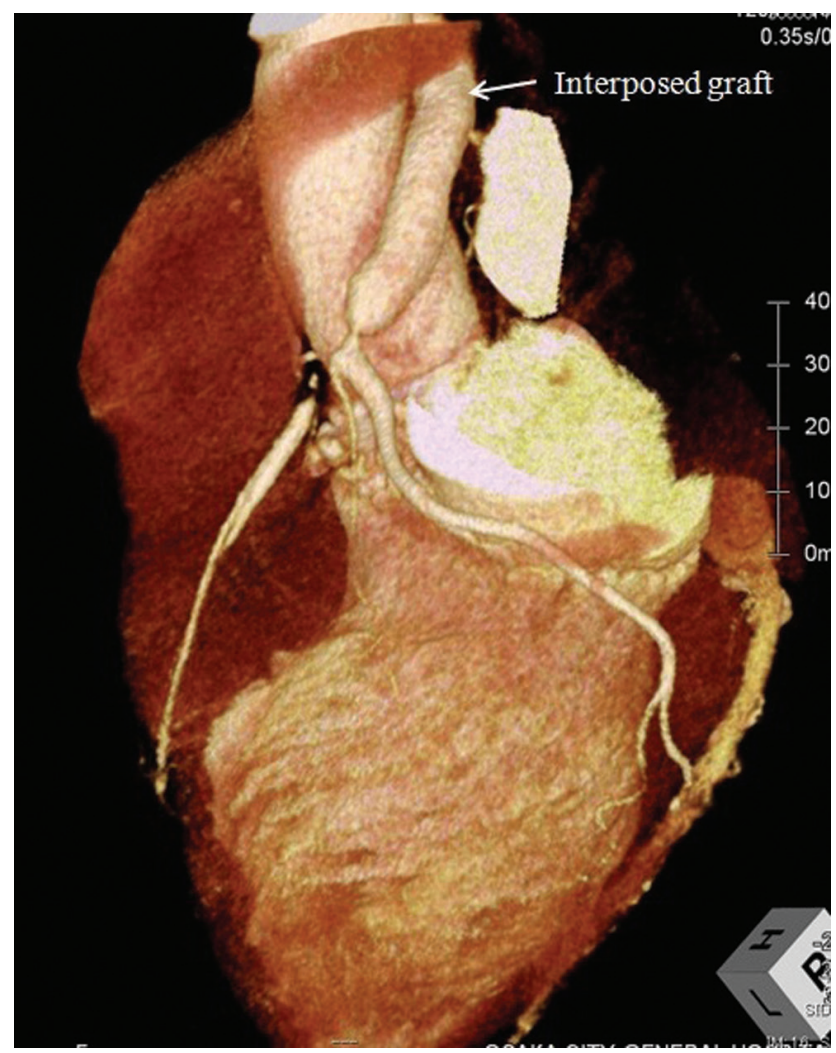

Fig. 1 Computed tomographic coronary angiography (CTCA) showed severe stenosis between the left coronary ostium (LCO) and the interposed graft as well as proximal stenosis of the left anterior descending artery (LAD).

re-reconstructed by the button technique. Postoperative computed tomographic coronary angiography (CTCA) showed no stenosis between the interposed graft and the LCO. Twelve months after surgery, CTCA revealed severe stenosis between the interposed graft and LCO and proximal stenosis of the left anterior descending artery (LAD) (Fig. 1). CABG to the LAD and left circumflex coronary artery (LCX) via a left thoracotomy was performed because pectus excavatum would prevent harvest of the left internal thoracic artery (LITA) through re-median sternotomy (Fig. 2) and to avoid potential injury during re-sternotomy. The proximal part of the LITA was easily accessed under an anterolateral incision at the fifth intercostal space and could be dissected proximally to the first intercostal space. The LITA was transected at the level of the fifth intercostal space as the distal LITA was strictly adhered. The LITA-radial artery (RA) composite graft was made for extension of the conduit and was anastomosed to the LAD by the off-pump technique. The pectus excavatum and adhesion of the
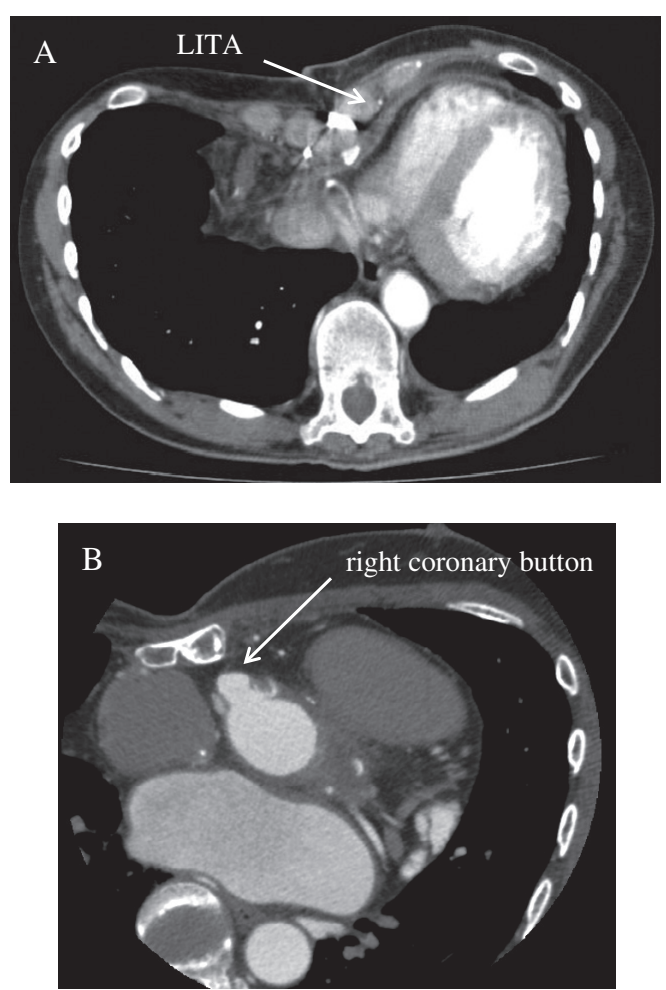

Fig. 2 (A) Pectus excavatum would prevent harvest of the left internal thoracic artery (LITA) through re-median sternotomy. (B) The sternum was tightly adhered to the heart. The right coronary button was located very close to the sternum.

heart prevented anterior shift and rotation of the heart. Thus, cardiopulmonary bypass was instituted to obtain good exposure of the posterolateral branch of the LCX. The residual RA graft, which was proximally anastomosed to the descending aorta, was anastomosed to the posterolateral branch by an on-pump beating technique. The patient became free of symptoms and is now in good condition.

\section{Discussion}

Anastomotic complications of the interposed coronary graft after the Bentall procedure is a rare but life-threatening complication. Sekine, et al. reported a patient with stenosis between a Dacron interposed graft and the coronary artery and supposed that the cause of the stenosis was intimal proliferation caused by turbulent blood flow. ${ }^{2)}$ On the other hand, Nakahira, et al. reported favorable outcomes without any complications regarding the interposed graft during follow-up after the modified 
Bentall procedure using Svensson's modification. ${ }^{3)}$ In this case, we re-reconstructed the aortic root using Svensson's modification because the LCO was tightly bound to the surrounding tissue. We believe that the stenosis of both the LCO and proximal LAD may have resulted from thrombosis secondary to turbulent blood flow in the interposed graft because this patient received anticoagulation alone before $\mathrm{CABG}$. Therefore, we recommend antiplatelet therapy, in addition to anticoagulation after the modified Bentall technique when using a long interposed graft to the coronary artery.

The left thoracotomy approach is a well-known technique in cardiac surgery, and was also reported being useful for cardiac operations in patients with pectus excavatum. ${ }^{4,5)}$ In this patient, who underwent a redo Bentall procedure, the right coronary artery as reconstructed by the button technique was very close to the sternum, which made repeat median sternotomy much more dangerous. Moreover, the LITA could not be dissected via repeat median sternotomy because of the pectus excavatum. Consequently, this patient was a good candidate for CABG through a left thoracotomy, from which both the LAD and the LCX could be accessed, and the LITA could be easily harvested although we experienced problematic exposure of the proximal LCX with the off-pump technique as the pectus excavatum and adhesion of the heart prevented anterior shift and rotation of the heart.

\section{Disclosure Statement}

None declared.

\section{References}

1) Svensson LG. Approach for insertion of aortic composite valve grafts. Ann Thorac Surg 1992; 54: 376-8.

2) Sekine S, Abe T, Seki K, et al. Dacron coronary graft obstruction after composite graft replacement of aortic root. Ann Thorac Surg 1995; 60: 1123-5; discussion 1125-6.

3) Nakahira A, Shibata T, Sasaki Y, et al. Outcome after the modified Bentall technique with a long interposed graft to the left coronary artery. Ann Thorac Surg 2009; 87: 109-15.

4) Pratt JW, Williams TE, Michler RE, et al. Current indications for left thoracotomy in coronary revascularization and valvular procedures. Ann Thorac Surg 2000; 70: 1366-70.

5) Choghari C, Heymans O, Geens M, et al. Left thoracotomy for coronary bypass in a patient with pectus excavatum. Ann Thorac Surg 1996; 62: 1182-3. 